\title{
CS preexposure: Latent inhibition and Pavlovian conditioning of heart rate and eyeblink responses as a function of sex and CS intensity in rabbits
}

\author{
LINDA L. HERNÁNDEZ, SHIRLEY L. BUCHANAN, and D. A. POWELL \\ Neuroscience Laboratory, Wm. Jennings Bryan Dorn Veterans' Hospital \\ Columbia, South Carolina 29201 \\ and Department of Psychology, University of South Carolina, Columbia, South Carolina 29208
}

\begin{abstract}
Male and female rabbits received Pavlovian conditioning in which a $1,216 \cdot \mathrm{Hz}$ tone served as the CS and a 3-mA paraorbital electric shock train served as the US. Eyeblink (EB) and heart rate (HR) CRs were assessed. Half of the animals received prior exposure to the CS, while half were restrained in the chamber for a similar length of time but did not receive prior CS exposure. Different groups of each sex received three different CS intensities including 60,75 , and $90 \mathrm{~dB}$ (SPL) during both preexposure and conditioning. The results revealed that latent inhibition of the EB CR occurred only at the intermediate CS intensity, as indicated by a significant impairment of EB conditioning in this group. However, the magnitude of the decelerative HR CR was attenuated by prior CS exposure at all three CS intensities. Females showed faster EB conditioning than males, but latent inhibition occurred in both sexes. These results suggest that somatomotor and autonomic systems are affected differently by prior CS exposure.
\end{abstract}

Lubow (1973) termed the presentation of a conditioning stimulus (CS), unpaired with an unconditioned stimulus (US) prior to conditioning training, "latent inhibition." Such a procedure usually results in a retardation of classical conditioning (Solomon, Brennan, \& Moore, 1974); organisms preexposed to the CS reveal deficits compared with nonexposed animals when later tested in a classical conditioning situation. The present study examined the effects of CS intensity on latent inhibition of classically conditioned heart rate $(\mathrm{HR})$ and eyeblink (EB) responses in the rabbit.

Previous experiments investigating the effects of CS intensity on latent inhibition have yielded mixed results. Crowell and Anderson (1972) found latent inhibition of the CER in rats to be a direct function of CS intensity. Schnur and Lubow (1976) also found that latent inhibition of the CER was a direct function of CS intensity provided that the CS was of the same intensity during acquisition as during preexposure. However, Lubow, Markman, and Allen (1968) found no effect of CS intensity on latent inhibition of the rabbit's pinna response, and Solomon et al. (1974) reported similar negative results in a classical conditioning experiment in which the nictitating membrane reflex was examined. In the same

This research was supported by VA Institutional Research Funds. Request reprints from D. A. Powell, Neuroscience Laboratory, Wm. Jennings Bryan Dorn Veterans' Hospital, Columbia, South Carolina 29201. A preliminary report of the present study was made at the Annual Meeting of the Society for Psychophysiological Research, Cincinnati, Ohio, October 1979. paper, however, conditioning deficits were found with CS preexposure to a $75-\mathrm{dB}$, but not a $95-\mathrm{dB}$, tone.

In the present experiment, we studied the effects of preexposure of CSs of different intensities on both somatomotor (i.e., eyeblink) and autonomic (i.e., heart rate) conditioning in the rabbit to see if differences obtained in prior studies could be related to the differential involvement of autonomic systems in different conditioning situations. The studies in which a direct relationship between stimulus intensity and latent inhibition was found involved the CER paradigm, which would be more likely to reflect conditioning of autonomic responses than more punctate responses such as the nictitating membrane and pinna responses. Possible differential sensitivity to CS preexposure by autonomic and somatomotor response systems might explain discrepancies in the results of prior studies. Since we had previously found that female rabbits showed faster tonic HR and smaller HR CRs than males (Buchanan, Hernández, \& Powell, 1979), CS preexposure was also studied as a function of sex.

\section{METHOD}

\footnotetext{
Subjects

Seventy-two experimentally naive male and female New Zealand albino rabbits (Oryctolagus cuniculus), obtained locally, were used as subjects. They were approximately 6 months old at the beginning of the experiment and weighed $2-3 \mathrm{~kg}$ each. The animals were housed in same-sex pairs in a room maintained on a 12-h (7 a.m./ 7 p.m.) light/dark cycle at a constant temperature $\left(20^{\circ} \mathrm{C}\right)$ and humidity. Food and water were available ad lib.
} 


\section{Apparatus}

Behavioral testing occurred during daylight hours in ventilated sound- and light-attenuating animal enclosures equipped with overhead speakers for delivery of auditory stimuli. During testing, each subject was restrained in a standard Plexiglas rabbit restrainer (Gormezano, 1966). All experimental events were controlled from an adjoining room by a minicomputer, which also collected and summarized the behavioral data from a Grass polygraph. Corneoretinal potential (EB) and electromyographic (EMG) recording electrodes were connected to wide-band preamplifiers and integrators, which were calibrated so that a $1-\mathrm{mm}$ pen deflection corresponded to a $100-\mu \mathrm{V}$ potential across the recording electrodes. Polygraph outputs were integrated over the CS interval (24 samples per channel for each CS interval), and the maximum amplitude of EB and EMG responses (in millimeters) and the latency from CS onset data were stored for subsequent analysis. Heart rate data were sampled eight times per half-second interval, averaged for 1-sec blocks, and stored for later analysis.

\section{Procedure}

Half the animals were male and half female. Within each sex, the subjects were randomly assigned to one of six subgroups formed by the factorial arrangement of two preconditioning exposure conditions (viz., exposed or not exposed) and three CS intensities $(60,75$, or $90 \mathrm{~dB}$ SPL).

On the first 2 days of testing, half of the animals (the "PRE" group) in each CS intensity condition received 100 nonreinforced presentations of the to-be-conditioned CS, which was a $500-\mathrm{msec}$ $1,216-\mathrm{Hz}$ square-wave tone at the appropriate intensity. The intertrial interval was $120 \mathrm{sec}$. The remaining animals (the "SIT" group) were treated in an identical manner except that the to-beconditioned CS was not presented. On Day 3, each animal in the PRE group received 32 further tone-alone trials, and the SIT group was further adapted to the chamber cues. One hundred tone-shock pairings followed during Session 3 for both the PRE and SIT groups. During these trials, all animals were exposed to the appropriate tone CS, immediately followed by a 500-msec 3.0-mA ac paraorbital shock train, which served as the US. The latter was delivered through Michel wound clips chronically implanted in the skin approximately $1 \mathrm{~cm}$ above and below the eye. The shock was omitted on four test trials in each session, during which HR was measured. During conditioning, the intertrial interval remained constant at $120 \mathrm{sec}$. On Days 4 and 5 of training, all animals received 100 conditioning trials, as above. Thus, each subject received a total of 300 conditioning trials preceded by (for the PRE group) 232 nonreinforced presentations of the to-be-conditioned CS or (for the SIT group) 232 trials during which data were collected but no stimuli, other than those associated with the chamber itself, were presented.

The EB response-consisting of eyelid closure, nictitating membrane extension, and/or eyeball retraction (see VanDercar, Swadlow, Elster, \& Schneiderman, 1969) - was measured on each trial by stainless steel electrodes inserted underneath the eyelids. A conditioned response was defined as a response occurring during the CS interval and exceeding a $200-\mu \mathrm{V}$ change from baseline. Trials on which the EB response was not at baseline at the beginning of the CS interval were not included in the data analysis. EMG was recorded on each trial from stainless steel insect pins acutely inserted into the neck muscles, and, like the EB response, was defined as a $200-\mu \mathrm{V}$ change from baseline during the CS interval. Again, trials in which EMG was not at baseline at the beginning of the CS interval were not included. This procedure resulted in discarding less than $1 \%$ of the EMG or EB data. HR was recorded during four preselected nonreinforced "test" trials from stainless steel safety pins inserted subcutaneously over the right shoulder and left flank. Mean HR was measured in beats per minute for four 1-sec blocks preceding and eight 1-sec blocks following the beginning of the CS interval.

The total number of EB responses, percent trials on which EB and EMG responses occurred, and mean EB and EMG ampli- tudes and EB latency were calculated for each subject for each preexposure and acquisition session. Data for the preconditioning and acquisition sessions were treated separately. Each dependent measure was assessed by separate mixed-design ANOVAs (group $\times$ CS $\times$ sex $\times$ session $\times$ subject). In addition, the total number of acquisition trials required to reach an acquisition criterion of five consecutive EB responses (trials to criterion, TTC) was calculated and subjected to separate between-subjects ANOVAs (group $x$ CS $\times$ sex $\times$ subject). Post hoc application of Duncan's multiple range test was used to determine the sources of significant $F$ ratios. The HR data were analyzed in a similar fashion. Mean baseline HR for each test trial was calculated as the average HR (in BPM) for the four 1-sec blocks preceding CS onset. The HR CR was then calculated as the mean HR associated with each post-CS second. These data were transformed to change-from-baseline scores by subtracting each post-CS second from the mean preCS HR score. These change-from-baseline scores were subjected to mixed-design ANOVAs (group $\times$ CS $\times$ sex $\times$ session $\times$ block $\times$ subject); again, data from the preconditioning and acquisition sessions were treated separately.

\section{RESULTS}

\section{Eyeblink Conditioning}

Figure 1 shows mean trials to reach a criterion of five consecutive EB CRs (top) and total number of CRs (bottom) as a function of sex and CS intensity. There was a significant increase in number of EB CRs and a significant decrease in trials to criterion as CS intensity increased $[\mathrm{F}(2,60)=15.5, \mathrm{p}<.0001$, and $F(2,60)=13.11, p<.0001$, respectively]. Post hoc

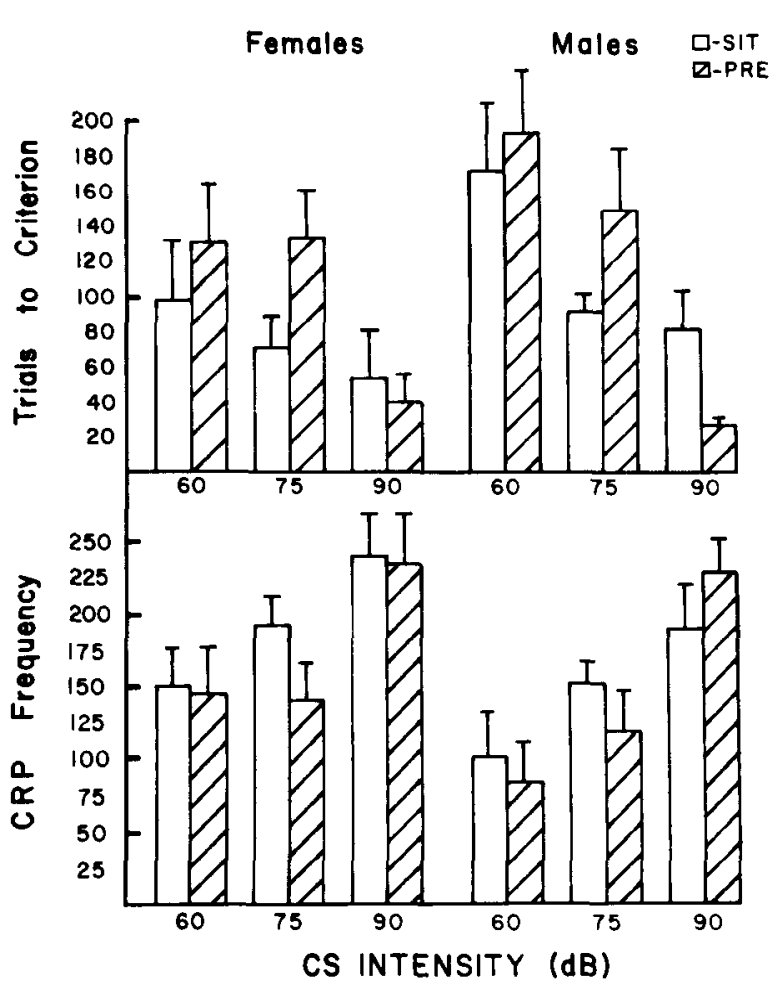

Figure 1. Trials to criterion (top) and total number of CRs (bottom) of male and female rabbits either exposed or not exposed to three different $\mathrm{CS}$ intensities prior to classical conditioning. 
tests revealed that animals trained to the 60- and 75-dB CSs did not differ significantly on these measures, whereas the 90-dB group showed more rapid conditioning. Females exhibited more EB CRs and required fewer trials to reach criterion than did males $[F(1,60)=6.59, \mathrm{p}<.01$, and $\mathrm{F}(1,60)=4.27, \mathrm{p}<.05$, respectivelyl. The overall differences between the SIT and PRE groups were not significant for any of the EB measures. The group $\times$ CS interaction for TTC approached but did not quite reach statistical significance $[F(2,60)=2.89, p=.06]$. However, post hoc tests, as well as planned ANOVAs performed on the TTC data from the three CS intensity groups, separately showed that there was a significant $(p<.05)$ retardation of conditioning produced by preexposure, but only in the group preexposed to the $75-\mathrm{dB}$ tone; thus, preexposure of the 60- and 90-dB CSs did not produce reliable latent inhibition of the eyeblink response. No significant differences between groups were observed for the other EB measures. Too few EMGs were recorded during acquisition to allow for a valid analysis of the data.

\section{Eyeblink, Preconditioning}

During the preconditioning phase of the study, a small number of EB responses were recorded from most subjects in both groups during tone presentation. Analysis of the total number of EB responses during preexposure showed that more EB responses occurred in the PRE than SIT groups $[F(1,60)=8.94$, $\mathrm{p}<.01$ ]; this finding was obtained during both 75 and 90-dB PRE conditions, whereas the 60-dB PRE animals did not differ significantly from the SIT controls $[F(2,60)=3.64, p<.05$, for the group $\times C S$ interaction]. Also, males tended to show more "spontaneous" EB responses than did females $[\mathrm{F}(1,60)$ $=3.97, \mathrm{p}<.05]$, and the amplitude of the EB responses during preexposure tended to be greater in males $[F(1,60)=3.55, p=.05]$ than in females. Too few EMG responses were again recorded from any group during preexposure to allow for a meaningful analysis of the data.

\section{Heart Rate Conditioning}

The HR data obtained during conditioning are shown in Figure 2. During conditioning, the HR CR was a deceleration; the magnitude of this response was greater for the SIT groups, indicating latent inhibition of the HR CR. However, these differences were most pronounced during the first session and decreased thereafter. The ANOVA performed on these data revealed that the group main effect as well as the group $\times$ sessions interaction were significant [group: $F(1,60)=3.87, p<.05$; group $\times$ sessions: $F(2,12)=7.68, p<.001]$. These findings thus suggest that latent inhibition occurred in the HR response system, but its effects were most dramatic early dur-

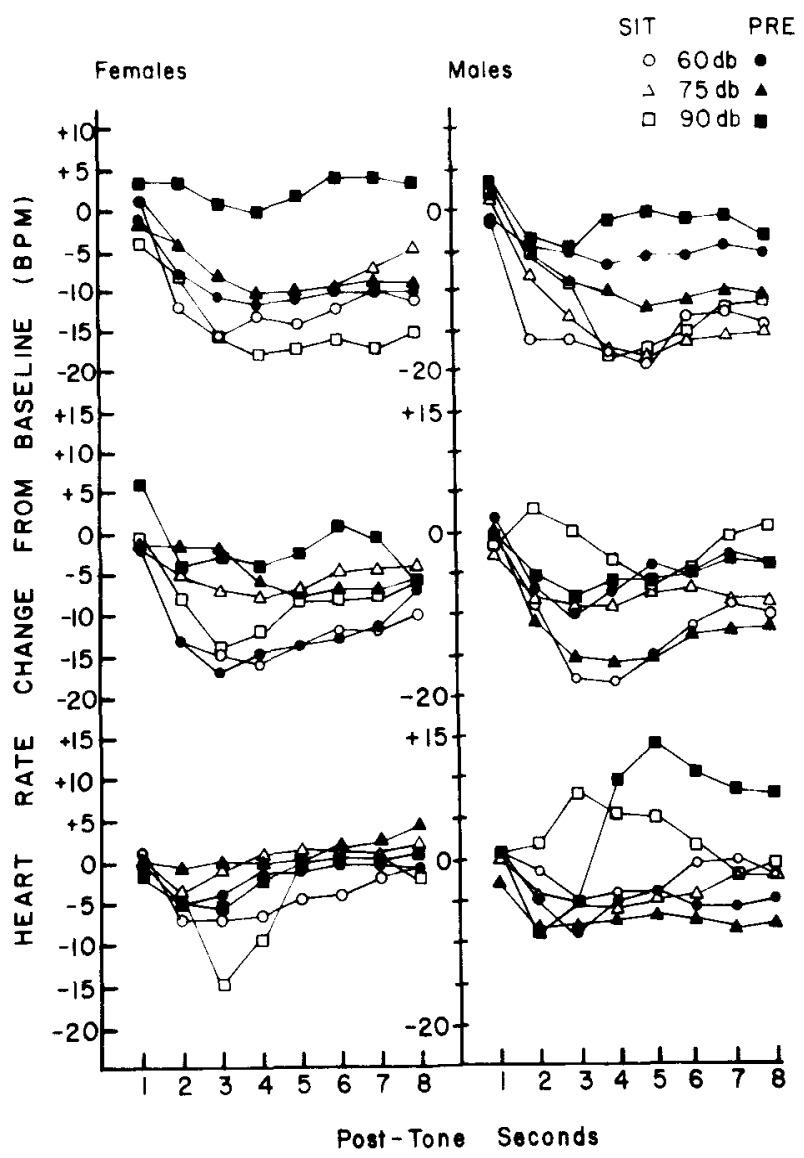

Figure 2. Heart rate changes from pre-CS baseline of male and female rabbits either exposed or not exposed to three different CS intensities prior to classical conditioning training. Data are shown for three consecutive daily acquisition sessions, from top to bottom.

ing conditioning and had essentially disappeared by the 2 nd and 3rd days of training. As Figure 2 also shows, the HR CR became less decelerative over acquisition sessions $[\mathrm{F}(2,120)=26.82, \mathrm{p}<.0001]$.

Figure 2 also indicates that stimulus intensity affected the magnitude of the $\operatorname{HR} \operatorname{CR}[F(2,60)=3.19$, $\mathrm{p}<.05]$. Thus, in general, the HR response to the 90-dB tone was smaller than that observed in response to the 60- and $75-\mathrm{dB}$ tones. This finding was especially true of the PRE animals that had been previously exposed to this tone and appeared to be more characteristic of males than females. Moreover, in the males, this relatively small bradycardiac CR changed during training to rather large HR accelerations in both the SIT and PRE groups. Although female subjects also showed an attenuation of HR CR magnitude at the 90 -dB CS intensity, overall it was somewhat smaller than that of the males. These differential changes over sessions as a function of sex and preexposure conditions produced two significant complex interactions, that is, CS $\times$ sex $\times$ session $\times$ 
block $[\mathrm{F}(28,840)=1.73, \mathrm{p}<.01]$ and group $\times \mathrm{CS} \times$ sex $\times$ session $\times$ block $[F(28,840)=1.84, p<.001]$.

\section{Heart Rate During Preconditioning}

Relatively large HR decelerations also occurred during preexposure of the CSs in the PRE groups. These data are shown in Figure 3, which depicts the mean HR change of both the SIT and PRE animals during the three sessions of CS preexposure. These HR changes represent the cardiac component of the orienting reflex (OR) as defined by Sokolov (1963), since they occurred in response to nonreinforced tones. Figure 3 shows that the cardiac OR consisted of bradycardia beginning within $2 \mathrm{sec}$ following CS onset and persisting throughout the 8-sec post-CS period. This response was somewhat greater to the 90- $\mathrm{dB}$ tone and appears to have habituated during the second and third preconditioning sessions. However, it actually became accelerative for the $90-\mathrm{dB}$ group during the second session. ANOVAs revealed these differences to be significant: group $[F(1,60)$ $=42.77, \mathrm{p}<.0001] ;$ group $\times \mathrm{CS} \times$ block $[\mathrm{F}(14,420)$ $=2.02, \mathrm{p}<.02)$; session $[\mathrm{F}(2,120)=4.62, \mathrm{p}<.02]$; group $\times$ session $[F(2,120)=8.38, p<.001]$; group $x$ session $\times$ block $[F(14,840)=2.41, p<.003]$; and $\mathrm{CS} \times \operatorname{session}[\mathrm{F}(4,120)=4.68, \mathrm{p}<.002]$.

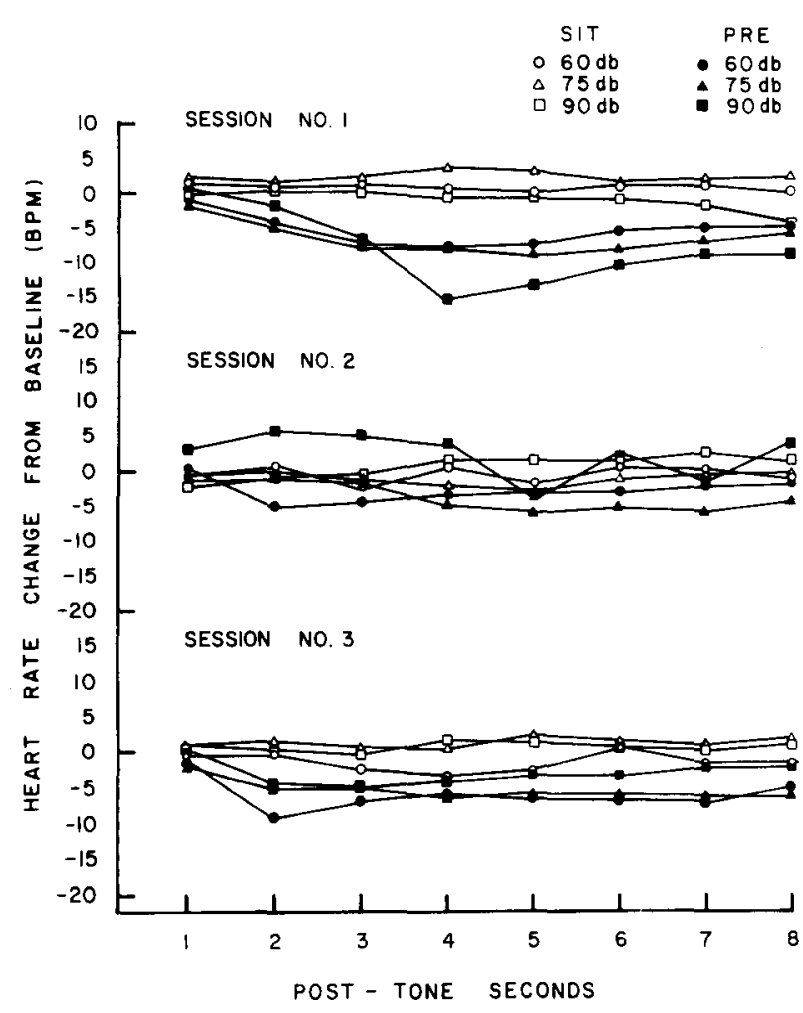

Figure 3. Heart rate change from a prestimulus baseline of rabbits exposed or not exposed to tones of three different intensities. Data are shown for three consecutive daily sessions.

\section{Baseline Heart Rate}

Analysis of the prestimulus (viz., baseline) HR data during both preconditioning and acquisition sessions showed a variety of significant effects. Since the HR CR has been shown in some cases to be at least partially determined by pre-CS baseline HR (Lacey, 1956), the HR CRs for each post-CS second were transformed by the method of Lacey into standard scores $(M=50, S D=10)$ in which the regression of baseline HR on change from baseline scores has been removed. ANOVAs were thus recomputed on these corrected scores. However, there were no differences between the results of these analyses and the analyses performed on the uncorrected scores, indicating that differences in the HR CR between the groups could not be attributed to differences in pre-CS baseline HR.

\section{DISCUSSION}

The results of the present experiment suggest that the effects of preconditioning CS exposure differ for somatic (EB) and autonomic (HR) responses. Pretraining exposure of low-intensity $(60-\mathrm{dB})$ or highintensity $(90-\mathrm{dB})$ tone CSs produced no reliable effects on subsequent eyeblink conditioning as assessed by any measure used in the present experiment. However, preexposure of an intermediate-intensity (75-dB) $\mathrm{CS}$ retarded eyeblink conditioning, as measured by trials to reach a preestablished criterion. In contrast with the EB results, CS preexposure produced reliable attenuation of the bradycardiac HR CR during the first 100 trial conditioning sessions at all three intensities, although no differences attributable to CS preexposure occurred during later training. Thus, while HR conditioning was a direct function of preconditioning CS intensity, demonstrable latent inhibition of the EB CR occurred only in animals preexposed to the 75-dB tone.

The deficits in the HR CR produced by CS preexposure are in contrast with those reported by Fitzgerald and Hoffman (1976), who found that 50 preconditioning CS exposures enhanced conditioning of a decelerative HR CR in rats. However, differences between the results of Fitzgerald and Hoffman (1976) and those of the present study are probably related to the larger number of CS preexposure trials used in the present study. Analogous results have been found for human electrodermal conditioning; up to 16 preexposures of the to-be-conditioned CS were found to enhance the magnitude of a conditioned skin-conductance response (Silver, 1973), whereas 50 or $100 \mathrm{CS}$ preexposures reduced the probability of occurrence of short-latency electrodermal changes during conditioning (Surwit \& Poser, 1974). This differential increase in responding after a few nonreinforced CS preexposures, followed by a de- 
crease after many nonreinforced CS presentations, is compatible with the dual process model of habituation and sensitization proposed by Groves and Thompson (1970).

In general, the results of the present study are not easily explained by current theories of latent inhibition, at least in their most recent formulations. Such theories generally attribute latent inhibition to "attentional deficits" resulting from preexposure to the $\mathrm{CS}$, that is, to the CS losing either "salience" or "associability" through a process resembling habituation (Lubow, Schnur, \& Rifkin, 1976; Mackintosh, 1975; Maltzman, Raskin, \& Wolff, 1979; Rescorla \& Wagner, 1972). The conditioned attention theory of Lubow et al., which basically states that the "attentional response" elicited by the first CS presentation is maintained by the US and, conversely, is lost by nonreinforced CS preexposure, is not inconsistent with the present HR results if it is assumed, as suggested by others (e.g., Maltzman et al., 1979), that this response is in fact an OR that eventually displays characteristic habituation. The finding that the $60-\mathrm{dB}$ tone produced little retardation of eyeblink conditioning compared with the $75-\mathrm{dB}$ tone is also consistent with conditioned attention theory (as well as other theories, e.g., Mackintosh, 1975; Rescorla \& Wagner, 1972), since the faster habituation characteristic of low-intensity stimuli (Groves \& Thompson, 1970 ) should also produce less latent inhibition. However, the lack of a latent inhibition effect on the EB response subsequent to preexposure of the $90-\mathrm{dB}$ tone is clearly inconsistent with a simple habituation model of latent inhibition, although it may not be inconsistent with more sophisticated models of the habituation process. For example, Sokolov's (1963) analysis of orienting and habituation as well as the dual process model of habituation proposed by Groves and Thompson (1970) suggest that habituation might be reduced or retarded in response to relatively intense stimulation. If habituation is the primary process underlying latent inhibition, such retardation should also reduce the magnitude of the latent inhibition produced by intense stimuli.

According to these models, the presentation of stimuli of high intensities elicits autonomic changes that might affect later learned behaviors in a fashion different from that associated with habituationnamely, a phenomenon similar to sensitization rather than habituation may be the end result of highintensity stimulation (Groves \& Thompson, 1970). The HR results of the present study, especially those obtained during the preconditioning phase, support these conclusions. Thus, although the initial HR response to the $90-\mathrm{dB}$ tone was pronounced bradycardia, this response reverted to tachycardia as a result of subsequent training. Tachycardia is obviously incompatible with the cardiac CR (which normally consists of HR decelerations) and thus produced $H R$ conditioning deficits compared with animals without CS preexposure. Previous studies have shown that relative increases in the HR CR are also positively correlated with EB conditioning (Powell \& Kazis, 1976). Thus, tachycardia would not be incompatible with the acquisition of the eyeblink response and indeed may reflect ongoing processes similar to "sensitization"' (Groves \& Thompson, 1970) that may facilitate increases in somatomotor responding. The finding that animals in the 90 - $\mathrm{dB}$ group showed more rapid EB conditioning than the 60- or $75-\mathrm{dB}$ groups, regardless of CS preexposure, supports this contention, since subjects in this group revealed relative HR increases as CRs. In this regard, it should also be noted that the cardiac "sensitization" response in the rabbit consists of HR increases (Powell \& Milligan, 1975). These considerations thus suggest that the effects of CS preexposure on conditioning depend not only on the characteristics of the CS and preexposure conditions (see Lubow et al., 1976), but also on the response being measured and its relationship to other ongoing behavior.

\section{REFERENCES}

Buchanan, S. L., Hernández, L. L., \& Powell, D. A. Agerelated changes in Pavlovian conditioning in rabbits: CNS correlates. Society for Neuroscience Abstracts, 1979, $5,3$.

Crowell, C. R., \& Anderson, D. C. Variations in intensity, interstimulus interval, and interval between preconditioning CS exposures and conditioning with rats. Journal of Comparative and Physiological Psychology, 1972, 79, 291-298.

Fitzgerald, R. D., \& Hoffman, J. Classically conditioned heart rate in rat following preconditioning exposure to the CS. Animal Learning \& Behavior, 1976, 4, 58-60.

Gonmezano, I. Classical conditioning. In J. B. Sidowski (Ed.), Experimental methods and instrumentation in psychology. New York: McGraw-Hill, 1966.

Groves, P. M., \& Thompson, R. F. Habituation: A dual-process theory. Psychological Review, 1970, 77, 419-450.

LACEY, J. I. The evaluation of autonomic responses: Toward a general solution. Annals of the New York Academy of Sciences, $1956,67,125-163$.

Lubow, R. E. Latent inhibition. Psychological Bulletin, 1973, 79, 398-407.

Lubow, R. E., Markman, R. E., \& Allen, J. Latent inhibition and classical conditioning of the rabbit pinna response. Journal of Comparative and Physiological Psychology, 1968, 66, 688-694.

Lubow, R. E., Schnur, P., \& Rifkin, B. Latent inhibition and conditioned attention theory. Journal of Experimental Psychology: Animal Behavior Processes, 1976, 2, 163-174.

Mackintosh, N. J. A theory of attention: Variations in the associability of stimuli with reinforcement. Psychological Review, 1975, 82, 276-298.

Maltzman, I., Raskin, D. C., \& Wolff, C. Latent inhibition of the GSR conditioned to words. Physiological Psychology, 1979, 7, 193-203.

Powell, D. A., \& Kazis, E. Blood pressure and heart rate changes accompanying classical eyeblink conditioning in the rabbit (Oryctolagus cuniculus). Psychophysiology, 1976, 13, 441-447.

Powell, D. A., \& Milligan, W. L. Effects of partial and con- 
tinuous reinforcement on conditioned heart rate and corneoretinal potential responses in the rabbit (Oryctolagus cuniculus). Psychological Record, 1975, 25, 419-426.

Rescorla, R. A., \& Wagner, A. R. A theory of Pavlovian conditioning: Variations in the effectiveness of reinforcement and nonreinforcement. In A. H. Black \& W. F. Prokasy (Eds.), Classical conditioning II: Current research and theory. New York: Appleton-Century-Crofts, 1972.

Schnur, P., \& LuBow, R. E. Latent inhibition: The effects of ITI and CS intensity during pre-exposure. Learning and Motivation, 1976, 7, 540-550.

Silver, A. Effects of prior CS presentations on classical conditioning of the skin conductance response. Psychophysiology, 1973, 10, 583-588.

Sokolov, E. N. Perception and the conditioned reflex. New York: Macmillan, 1963.
Solomon, P. R., Brennan, G., \& Moore, J. W. Latent inhibition of the rabbit's nictitating membrane response as a function of CS intensity. Bulletin of the Psychonomic Society, 1974, $4,445-448$.

Surwit, R. S., \& Poser, E. G. Latent inhibition of the conditioned electrodermal response. Journal of Comparative and Physiological Psychology, 1974, 86, 543-548.

VanDercar, D. A., Swadlow, H. A., Elster, A. E., \& SchneIderman, N. Nictitating membrane and corneoretinal transducers for conditioning in rabbits. American Psychologist, $1969,24,262-264$.

(Manuscript received June 19, 1980; revision accepted for publication May 26, 1981.) 